\title{
TRAINING
}

\section{Planning for coronary angioplasty: guidelines for training and continuing competence}

British Cardiac Society (BCS) and British Cardiovascular Intervention Society (BCIS) working group on interventional cardiology

Members of the working group: D J Parker (Chairman) (Immediate Past President, British President, British H H Gray (Secretary) (Honorary Secretary, British Cardiovascular Intervention Society); R Balcon (President, British Cardiac Society); J S Birkhead

(consultant cardiologist, Northampton General

Hospital);

R M Boyle

(consultant cardiologist,

York District Hospital);

I Hutton (Director of

Clinical Medicine,

Glasgow Royal

Infirmary);

L Parsons (Deputy

Director of Public

Health, East London

Health Authority);

M T Rothman

(Chairman, British

Cardiovascular

Intervention Society);

T R D Shaw (Honorary

Treasurer, British

Cardiovascular

Intervention Society).

Correspondence to:

Correspondence to:

Cardiac Unit, Southampton

Cardiac Unit, South

Southampton SO16 6 YD

Accepted for publication

5 December 1995
Since Grüntzig's first report of successful balloon dilatation of a human coronary artery in $1978^{1}$ there has been a rapid expansion in the number of interventional procedures undertaken worldwide and angioplasty is now established as one of the major treatments for patients with coronary heart disease. Additional coronary interventional technology has been developed such as stents, atherectomy, drill and laser devices, and catheter based diagnostic imaging techniques such as intravascular ultrasound and angioscopy are used increasingly. Guidelines for the selection of patients for these procedures have been detailed elsewhere. ${ }^{2}$ In the United Kingdom about $80 \%$ of angioplasty procedures are to a single vessel and $20 \%$ are multivessel for patients with stable or unstable angina. ${ }^{3}$ Encouraging results have recently been reported for the use of primary angioplasty in the treatment of acute myocardial infarction ${ }^{4-6}$ but its more widespread application is not yet established, partly because of the logistical difficulties and cost implications of delivering such a service at a local level. ${ }^{7}$ The need to develop such a service in the United Kingdom would have a major impact on planning for the future number of trained operators and volume of angioplasty procedures, and should be kept under regular review. Interventional cardiology now involves a complex range of manual and decision-making skills. We have considered how these skills may best be developed in the United Kingdom and the appropriate environment in which to practise them.

\section{Quality assurance}

The desirability of achieving high standards of care for patients undergoing interventional cardiological procedures is not contentious. However, attempts at defining the ideal environment in which these high standards may apply highlight some quite different approaches to the practice of intervention, not just between centres or between individual operators in the United Kingdom, but also between countries. The purpose of this document is to address these differences and try to define standards of practice for percutaneous coronary intervention in the United Kingdom, both for individuals and institutions. This will assist those involved in planning for future developments and is relevant to purchasers and providers of health care who will increasingly include measures of quality as well as cost and volume in contract agreements. It is not the intention of this document to be proscriptive; rather, it is an attempt to define a baseline standard of good practice that will carry as broad acceptance as possible and upon which future developments in interventional cardiology can be based.

\section{Audit}

Audit of casemix, procedural success, and complications and both in-hospital and six month post-procedural outcome must be the best way to assess quality of care for patients but such audit processes are time-consuming, require financial and manpower support to achieve, and currently do not exist. Also, they depend crucially on the active participation of individual centres and operators and their willingness to share their data locally and nationally. The British Cardiovascular Intervention Society (BCIS) has collected audit data of procedural outcome since 1988 but despite strenuous attempts to improve data collection several centres still fail to provide even simple information. Of the 53 centres undertaking percutaneous transluminal coronary angioplasty (PTCA) in the United Kingdom in 1993 four were unable to report the total number of procedures performed in their centre and $11(21 \%)$ were unable to report their mortality. ${ }^{8}$

We believe that the poor response of some centres is not due to their inherent reluctance to share data but to the lack of a system in place for the prospective collection of information and hence the dependence on retrospective data collection at the end of each year. 
With current data recording depending mostly on medical and nursing case notes this task of retrospective data collection is difficult, time consuming, and inherently inaccurate. For centres, operators, and purchasers to know more accurately what actually happens to patients undergoing PTCA we need much better audit processes, and for potentially helpful anonymous comparisons to be possible between centres, this data needs to be shared at a national level through annual returns to BCIS.

\section{MINIMUM DATA SET}

A Central Cardiac Audit Database Committee has recently been set up with Department of Health support to develop a minimum data set relevant to various specialist cardiac groups, such as interventionists, electrophysiologists, cardiac surgeons, paediatric cardiologists, and cardiac anaesthetists and this should be piloted in some centres in 1996. The data set will include pre-procedural, procedural, and post-procedural information. Pre-procedural patient data must be sufficient to allow risk stratification to be made. Procedural information will include the nature of the procedure, the status of the operator, the equipment used, and documentation of the result and any complications. Post-procedure information will initially concentrate on the patient's in-patient stay, although collection of out of hospital follow up data will also need to be developed. At present BCIS collects procedural data but only very limited clinical or follow up data and so longer term outcome is unknown, and risk stratification and comparisons between centres have been impossible. The RITA trial, ${ }^{9}$ which involved a number of United Kingdom centres and compared PTCA with coronary artery bypass grafting (CABG), has provided some long term information on outcome, although this was obviously based on a small sub-group of patients selected for their suitability for randomisation into the study.

Once the minimum data set and the methods by which the data can be collected are defined, adequate funds will need to be provided in each centre to allow efficient collection. This will probably require the local audit process to be costed and built into contracts with purchasers since central funding is unlikely. Individual operators should be responsible for collecting data relating to their own procedures and be prepared for them to be presented for peer review. One mechanism by which this might be achieved is for audit results to be presented regularly at a local level to those involved with PTCA, such as interventional and non-interventional cardiologists, cardiac surgeons, nursing, and technical and radiography staff and the data should be available to purchasers. All centres should submit annual returns to BCIS. We would welcome the participation of purchasers in such audit and some may consider it appropriate to include it as a condition in contracts.

Even if improved audit collection in future provides us with a more sophisticated means of setting standards this will take some years to evolve and at present we simply do not have the necessary data to set standards based on a firm scientific basis. Some would argue that we should therefore not attempt to define these standards at all until the auditing process is in place and returning reliable data. We disagree with this view. Purchasers of health care increasingly request guidelines so that clauses relating to quality can be built into their contracts with providers and if those involved in interventional cardiology do not help to define these standards, however much they may be based at present on professional judgement rather than scientific fact, then purchasers will draw up their own criteria. It is our view that these may be restrictive, less well informed, and may be unduly influenced by factors relating to cost containment. Also, attempting to set standards and emphasising the importance of audit should encourage all involved in interventional cardiology to audit their activities and through national data collection the audit information may assist centres in the process of bidding for appropriate local facilities, clinical audit, and funding for an appropriate workload. Additionally, a process of continuous audit will allow individual operators or a unit as a whole to recognise early when standards need local review.

\section{Standards for operators}

Coronary angioplasty is a skilled procedure with success and complication rates relating to the operator's expertise and judgement. It is obviously essential that those providing such a service to patients are properly trained and maintain their competence by continued practice. Intuitively, there must be a minimum number of procedures undertaken annually below which the necessary skills are not maintained. ${ }^{10}$ Most would accept this but setting a value to this minimum number engenders heated debate. In 1993 the Joint Task Force of the American Heart Association and American College of Cardiology (ACC/AHA task force) ${ }^{2}$ recommended 75 procedures per annum as the minimum required to maintain competence. Since that review was undertaken the technology associated with coronary angioplasty has become more complex, with much greater use of intracoronary stents.

In the United Kingdom a high percentage of operators undertake relatively few procedures annually and these low-volume operators argue, with some justification, that by careful case selection and with a realistic appreciation of their own expertise they can achieve a high level of success with a low number of complications and that far from being less safe they may have a lower absolute number of complications than the high volume aggressive PTCA operator who undertakes more complex procedures. This comparison obviously ignores the effect of different casemixes between operators. Also, lower volume operators argue that if the minimum number of procedures which they have to achieve is set above, and sometimes considerably above, their present activity they are left 
with a difficult choice. Either they are forced to abandon interventional cardiology, which for the very low volume operator may be appropriate, or they may feel pressurised into undertaking procedures which they consider either inappropriate or ones which they may feel less comfortable performing. This may consequently increase rather than decrease the frequency of their complications. Thus, setting a level for the absolute minimum number of procedures conducive to maintaining adequate skills is perhaps the most contentious of all issues relating to the setting of standards in interventional cardiology.

Having heard representations from many sources and taking account of the current provision of angioplasty services around the United Kingdom and the low volume of procedures undertaken by some senior operators it is our view that by 1996-97 any operator who is trained in angioplasty should be performing a minimum of 1-2 procedures per week and that this should normally amount to an annual total of not less than 60 procedures as primary operator. Consultants currently undertaking PTCA will have different past levels of experience but for the purposes of this document all consultants currently undertaking PTCA are included in the description "trained operator". At the end of each PTCA procedure a single person should be recorded as the primary operator, and this should be a person actively involved with the case and assuming principal responsibility for its outcome.

It is our view that ideally all trained operators should undertake more than the minimum to maintain competence and that strenuous efforts should be made by those operators currently undertaking low volumes to increase their experience and level of activity to at least 100 procedures per annum over the next three years. We recognise that improved audit data may allow more sophisticated standards to be set that rely less on mere volume of procedures, and it is vital that before these issues are reviewed again in three years, centres and individuals make a commitment to collecting and sharing relevant data. The Central Cardiac Audit Database Committee will assist in this process by establishing a minimum data set and offering advice to centres and individuals, and BCIS will continue to collect data from all centres undertaking interventional procedures.

\section{Standards for centres}

\section{CATHETER LABORATORIES}

A centre undertaking PTCA should have a cardiac catheter laboratory or laboratories, equipped with a physiological measurement system and full facilities for cardiopulmonary resuscitation, including an intra-aortic balloon pump. These facilities and an adequate number of trained nursing, technical, radiographic, and senior and junior medical staff must be available to provide a 24 hour service throughout the week, including weekends. The intervention laboratory and its staff should be fully operational within 60 minutes of being notified of urgent need.

High quality $x$ ray imaging equipment, preferably digital, that is capable of imaging the coronary arteries from all angles, including cranial and caudal angulation, must be provided. Image manipulation including freezeframe, zoom, and playback must be immediately available and a roadmapping facility is desirable. Radiation exposure should be kept to a minimum and good radiation protection should be provided for catheter laboratory staff as well as patients. ${ }^{11}$ The need for particular items of angioplasty hardware, such as balloons, guidewires, guiding catheters and stents often cannot be anticipated until a procedure is in progress and therefore it is vital that a full range of equipment is kept available at all times. As angioplasty hardware has a limited shelf life this implies that to be cost effective a centre must carry out a reasonable volume of procedures. The potential need to upgrade and extend equipment as advances are made needs to be anticipated and planned for.

\section{VOLUME OF PROCEDURES}

The skills of all staff and the cost efficiency of a centre can be improved by having adequate numbers of procedures undertaken each year. Too few procedures results in inexperience and is potentially dangerous. Hospitals which perform more PTCA procedures have lower referral rates for emergency $\mathrm{CABG}$ and shortterm mortality after the procedure, ${ }^{12-14}$ and this has been shown to be independent of differences in case mix. ${ }^{13}$ It is our opinion that each centre should have at least two and preferably three, competent trained operators so as to ensure continuity of service provision and that 200 PTCA procedures per annum should be regarded as the minimum that a centre should undertake. There is evidence that the number of complications following PTCA significantly diminishes with annual institutional case loads above 400 procedures, ${ }^{13}$ so centres undertaking fewer procedures should aim to increase their volume of activity as soon as possible. Since 10 of the 42 NHS centres undertaking PTCA in the United Kingdom in 1993 performed fewer than 200 procedures $^{8}$ (eight of these performed fewer than 150) we recommend that the minimum target of 200 per institution be achieved by the end of 1996-97. The minimum number for a centre should still be 200 per year even if trained operators undertake PTCA in other centres since support staff such as catheter laboratory nurses, technicians and radiographers, ward nurses, and junior doctors all need to experience a sufficient number of cases in their centre to ensure institutional competence. The results of individual operators can be improved by sharing experience with colleagues and we believe interventional centres should hold regular meetings to discuss cases.

Given that all those undertaking PTCA procedures accept that technical skills are required for the safe performance of interven- 
tion and that these skills require regular updating in the light of newly emerging technology we believe the United Kingdom should continue to concentrate interventional cardiology services in centres with relatively high volumes of procedures, using operators who maintain a high level of experience and offer a full range of interventional techniques, especially intracoronary stenting. If primary PTCA for acute myocardial infarction were to gain widespread acceptance as a treatment of choice then clearly angioplasty services will have to be developed much more widely than is currently the case. However, if this were to occur the potential numbers being undertaken could be considerable and so the volume of activity in almost any proposed new centre would be sufficient to maintain institutional and individual competence. Although the routine use of angioplasty in the management of acute myocardial infarction has yet to be established, exceptional circumstances may arise where angioplasty would be generally regarded as the treatment of choice and potentially life saving but the patient is considered either too unwell to be transferred to an angioplasty centre or the delay in making such a transfer is considered to be clinically unacceptable. We accept, as does the ACC/AHA task force, ${ }^{2}$ that in exceptional circumstances it may be appropriate for so called "salvage" angioplasty to be undertaken by a trained operator in a centre not routinely undertaking PTCA procedures. There are likely to be few such cases at present.

If potential new centres are likely to satisfy the recommendations contained in this document then they should be encouraged to develop because the United Kingdom requires a considerable increase in PTCA activity. However, a situation in which there is unplanned proliferation of PTCA in low volumes undertaken in district hospitals with coronary angiography facilities is one that is likely to be associated with sub-optimal results, a higher frequency of complications and almost certainly higher unit costs. We consider such a development to be undesirable.

\section{SURGICAL COVER}

The issue of surgical cover for PTCA procedures has been an emotive one, with on the one hand cardiac surgeons generally regarding it as mandatory and requiring it to be on the same site, and on the other some interventionists regarding surgical cover as unnecessary on or even off site. ${ }^{15}$ Although emergency CABG within the first 24 hours was required in only $2.0 \%$ (240 patients) of all reported procedures in the United Kingdom in $1993^{\circ}$ there is still a consensus that prompt access to emergency surgery, whether on site or off site, should still be available for all patients undergoing PTCA, other than the few who have been agreed not to require cover. Intracoronary stenting is not a panacea for abrupt coronary occlusion during PTCA. Although bail-out stenting can often salvage an otherwise failed angioplasty procedure and may avoid surgery the results of stenting in these circumstances compared with elective insertions are less predictable and where successful may be sub-optimal and merely allow the patient to be stabilised before transfer to the operating theatre. Of the 12364 PTCA procedures undertaken in the United Kingdom in 1993 a total of 11385 (92\%) were performed in the 45 centres with on site surgical cover and $979(8 \%)$ were undertaken in the seven centres with off site cover. ${ }^{8}$

Guidelines published by BCIS in $1992^{16}$ recognised that PTCA could be undertaken safely in centres without on site surgical cover but recommended that on site surgery was the strongly preferred option. Since 1992 several European centres have demonstrated good results from PTCA undertaken with off site surgical cover, but these have usually been centres with a high volume of procedures, undertaken by experienced operators, and offering short transfer times to off site surgery when this is required. It is unrealistic to extrapolate the results from these units to those undertaking relatively low volumes in the United Kingdom and expect the results to be the same. We consider that surgical cover is still necessary for PTCA procedures and that on site surgery remains the strongly preferred option.

A centre without on site surgical cover, but meeting the general standards of care outlined above and able to undertake at least 200 PTCA procedures per year must ensure that reliable arrangements are in place to allow the prompt transfer of a patient to a suitable operating theatre, cardiac anaesthetist, and cardiac surgeon. The covering surgeon should know that the PTCA procedure is being undertaken and an arrangement must exist with the local ambulance service for the immediate availability of an ambulance, fully equipped with resuscitation facilities, for emergency transfer of the patient when necessary. These arrangements should be explicit and agreed with purchasers and the ambulance service. We recommend that the journey time between the two hospitals should not exceed 30 minutes and that all centres, whether with on site or off site surgical cover, should be able to establish cardiopulmonary bypass within 90 minutes of the decision being made to refer the patient for emergency surgery. Time to emergency cardiopulmonary bypass should be included in the audit process.

\section{POST-PROCEDURE CARE OF PTCA PATIENTS}

The safety and success of angioplasty is not determined solely by the quality of the procedure itself. Appropriate case selection is an obvious example of an important preprocedure determinant of outcome, but postprocedure care is equally important. Centres undertaking PTCA should have a sufficient throughput to ensure that junior medical and ward nursing staff become experienced in the observation and management of patients, such that the management of post-procedure chest pain or femoral complications is well understood. Facilities should be available for elec- 
trocardiographic and pressure monitoring for patients after angioplasty. Femoral artery sheath removal is associated with vasovagal and bleeding complications, both of which may jeopardise the outcome of an angiographically successful PTCA procedure, and staff should have experience and be trained in the avoidance of complications and their management when they do occur. There should be immediate and 24 hour access to haematological, biochemical, and blood transfusion laboratories and vascular surgical advice and intervention should be readily available.

The overall quality of care of patients undergoing PTCA is dependent on all staff dealing with PTCA patients being experienced, and on clear nursing and medical management protocols for dealing swiftly and efficiently with the complications of PTCA that inevitably occur in some cases. Achieving high quality of care is the overriding objective, but in an increasingly litigious world the medicolegal implications of failing to provide this comprehensive approach to care should also be considered most carefully.

If PTCA procedures are undertaken by an operator at a site remote from their normal base hospital the visiting operator should either be available personally for 24 hours after the procedure to offer immediate cover in the event of a post-procedure complication or should have ensured the same availability of another fully trained consultant who is based at the hospital where the procedure was performed.

\section{Training and accreditation \\ TRAINEES}

The new Specialist Registrar training to be introduced following the Calman recommendations consists of five years Basic Training and an Advanced 6th Year. ${ }^{17}$ In the basic years the individual is expected to have assisted at 25 PTCA procedures but training in angioplasty as a specialty does not start formally until the advanced year. With the likelihood of there being a limited number of consultant interventionist posts falling vacant each year, only a few specialist trainees will be required in the advanced year. No indication has been given as to whether the number of advanced training posts in PTCA will be limited by external allocation and accreditation or whether it will be left to individual trainees to decide whether the training in PTCA in their particular centre would be of sufficient quality and the possibility of a consultant vacancy in future sufficiently high to commit themselves to specialist training. Formal manpower planning may be necessary and it seems inevitable that a selection process will be needed to identify those individuals most naturally suited to undertaking interventional procedures. For planning purposes training posts should not be counted as providing any service commitment.

Defining the minimum number of PTCA procedures that constitutes an acceptable training in angioplasty is difficult because individuals learn at different rates and case selec- tion and available facilities will differ between centres. However, the new training recommendations state that the trainee having started advanced training in PTCA must undertake at least 100 PTCA procedures in the advanced year, 50 of which must be as principal (first) operator. Experience in previous years cannot be aggregated with those in the advanced year. It is not uncommon for more than one operator to be involved in PTCA procedures and it can sometimes be difficult to define clearly the principal operator. However, for the purposes of training it should be left to the trainer to determine when a trainee has been the principal as opposed to assistant (second) operator. The trainee must be familiar with the catheter laboratory and angioplasty equipment and understand the radiation implications of PTCA procedures and the means by which radiation exposure can be minimised. In addition to performing PTCA effectively the trainee should be directly involved in the subsequent care of patients on the ward and after discharge from hospital, and the auditing of results for the centre as a whole. The individual should be involved in discussions concerning case and equipment selection and must keep abreast of the literature on PTCA. The trainee is expected to maintain a log book of all catheter laboratory procedures throughout training and should spend at least four days each year attending appropriate educational meetings, which in the case of an advanced trainee in PTCA would be at meetings involving interventional cardiology.

\section{TRAINERS}

Operators undertaking the training of those in the advanced (6th) year of their specialist education in cardiovascular medicine should be involved in the selection of potential trainees and should themselves undertake a minimum of 125 procedures personally each year, in addition to the 50 procedures required by the trainee. Trainers should be experienced angioplasty operators having undertaken at least 500 cases personally and should work in centres offering a full range of interventional procedures. Only a minority of existing operators will be required to undertake training if the number of junior staff being trained in PTCA falls as greatly as expected as a result of the new training recommendations. The trainer will be responsible for confirming that the trainee has completed an interventional training year satisfactorily: the Specialist Advisory Committee in Cardiology of the Royal College of Physicians should consider establishing a formal accreditation procedure.

\section{The future}

DEMAND FOR PTCA IN THE UNITED KINGDOM It is difficult to define the true needs of the population for coronary revascularisation procedures such as PTCA and CABG surgery because of a lack of good epidemiological data. The only data available from which an assessment of need might be calculated are 
standardised mortality ratios for coronary heart disease, which correlate poorly with the referral rates for PTCA and CABG in the United Kingdom. There are considerable geographical variations in the United Kingdom for both the prevalence of coronary heart disease and the provision of revascularisation procedures. ${ }^{18}$ Unmet demand for intervention procedures, as measured by waiting lists, may be used as a surrogate indicator of population need but is likely to underestimate considerably the true need. If long waiting lists were an indicator merely of a backlog of unmet demand rather than continuing need then the number of patients on waiting lists should gradually fall as the provision of services improves. Far from doing this, the number of patients waiting for intervention is increasing which strongly suggests continuing population need. The Government has recognised this need by the importance it has placed on coronary heart disease in the Health of the Nation document. ${ }^{19}$

The United Kingdom undertakes relatively few revascularisation procedures compared with other European countries and with the $\mathrm{USA}^{20}$ and this is another reason for believing that the United Kingdom does not meet the true needs of its population. In 1994 the British Cardiac Society published guidelines for the provision of cardiac services ${ }^{21}$ and indicated a target of 400 PTCA and 600 CABG procedures per million to be achieved by 1996-97. It was recognised that the results of clinical trials, changing technologies, and the possible extension of angioplasty to acute myocardial infarction would increase the need for these procedures well above the recommended target, and may alter the balance between them. It was therefore emphasised that these targets represented a conservative estimate of true population need and that, because of the number of existing patients with coronary heart disease, the target figures were likely to increase for many years despite any fall in the incidence of ischaemic heart disease. In 1992 the United Kingdom undertook a total of 203 PTCA procedures per million population. ${ }^{8}$ Based on returns to BCIS this rose to 230 per million in 1993 and from preliminary data is projected to have risen to 250 per million in $1994 .^{8} \mathrm{~A}$ significant expansion of the workload is therefore required if the provision of angioplasty services is to meet the true needs of the population more closely.

\section{FUTURE NEED FOR INTERVENTIONAL} CARDIOLOGISTS

For future planning purposes if we use an average annual number of 100 procedures per operator and the conservative target figure of 400 per million population (total $=23000$ procedures) the United Kingdom requires 4 operators per million of the population served or a total of 230 trained principals undertaking PTCA. From returns to BCIS, the 12364 procedures undertaken in the United Kingdom in 1993 were performed by 170 principal operators, ${ }^{8}$ representing a mean volume of 72 procedures per operator. If existing lower volume operators increase their activity the UK could achieve the target number of 400 procedures per million with only a small expansion in the number of trained operators. The number of operators would not have to be expanded greatly even if low volume operators continue at their present level. This calculation does not allow for procedures undertaken by those in training.

FUTURE NEED FOR INTERVENTIONAL CENTRES For the target figure of 400 PTCA per million population to be achieved many more procedures will need to be undertaken than at present. To achieve this increase one might encourage new centres to develop, or expand the activities of existing ones. These options are not mutually exclusive and if new centres can fulfil the guidelines outlined in this document and offer the realistic prospect of gradually expanding their activities then their development should be encouraged and supported. It seems logical that with the development of local cardiac catheterisation facilities, diagnostic cardiac catheterisation will increasingly be undertaken in district hospitals and proportionately fewer in the present interventional centres. The reduction in diagnostic workload of the interventional centres should allow relatively more time for angioplasty procedures.

Most (86\%) units currently undertaking PTCA in the United Kingdom are in cardiac surgical centres, each serving populations of around 1.5-3.0 million people, and these surgical centres undertook $92 \%$ of all the PTCA procedures in $1993 .^{8}$ These centres would perform 600-1200 PTCA procedures per year if all suitable patients were referred to these centres and the target figure of 400 per million were achieved. Also, centres with surgery on site already have trained staff offering 24 hour emergency catheter laboratory and surgical cover and offer regular contact between angioplasty operators and surgical colleagues for discussion of cases.

Where diagnostic coronary arteriography is carried out in the district hospitals it is important that a close liaison is developed between the cardiologist undertaking these diagnostic procedures and the centre to which those patients selected for revascularisation (PTCA or CABG) would be referred. This is particularly relevant to coronary intervention where the indications for procedures, the range of technologies to undertake these procedures and the management of patients subsequently, have changed so rapidly. A good liaison between the referring cardiologist and the interventionists and surgeons will help to ensure that the appropriate revascularisation procedure is chosen. We suggest that those undertaking diagnostic angiography should have regular clinical meetings which involve both an interventional cardiologist and cardiac surgeon. Those undertaking revascularisation procedures should provide audited data of their results to their referring hospitals. 


\section{Further review}

With the emergence of new technologies and the possible extension of the role of PTCA in the management of patients with acute myocardial infarction, and in anticipation of improved audit data, it seems appropriate to review the recommendations in this report in three years time. The Council of the British Cardiac Society and the Council of the British Cardiovascular Intervention Society propose to establish a further working group in 1998-99.

\section{Conclusions}

The following recommendations are made:

1 Existing centres undertaking angioplasty should increase their activity, and the target figure of 400 PTCA procedures per million of the United Kingdom population should be achieved by the end of 1996-97, or immediately thereafter.

2 Angioplasty centres should be appropriately equipped to undertake PTCA safely and effectively and provide a reliable emergency service. They should have a minimum of two trained PTCA operators jointly undertaking a minimum of 200 procedures per year at that centre, and have regular meetings to share experience.

3 Angioplasty operators should ensure that where the need arises patients undergoing PTCA can receive immediate attention from a trained operator at any time until discharge from hospital.

4 Trained operators should undertake at least 1-2 PTCA procedures per week ( $>60$ procedures per year) to maintain competence, and those undertaking so few procedures should increase their activity over the next three years to more than 100 a year.

5 Trainers should have performed at least 500 procedures before formally training others and should undertake a minimum of 125 procedures a year to maintain accreditation as a trainer.

6 Surgical cover for PTCA procedures should be mandatory and on site cover remains the strongly preferred option. Where surgical cover is provided off site, this should be at a centre less than 30 minutes away by road. Whether provided on or off-site it should be possible to establish cardiopulmonary bypass within 90 minutes of the decision being made to refer the patient for surgery.

7 All operators and interventional centres should audit their activity and results, review these data locally with colleagues, and provide regular audit returns to the national database run by BCIS. This will allow future recommendations concerning standards to take more account of risk stratification and actual outcomes, and not place such emphasis merely on volumes of activity.

8 These recommendations should be reviewed in three years.

1 Grüntzig AR. Transluminal dilatation of coronary artery stenosis. Lancet 1978;i:263.

2 Ryan TJ, Bauman WB, Kennedy JW, et al. Guidelines for percutaneous transluminal coronary angioplasty.
Circulation 1993;88:2987-3007.

3 Cardiac interventional procedures in the United Kingdom during 1991. Hubner PJB on behalf of the British Cardiovascular Intervention Society. Br Heart $\mathcal{f} 1993 ; 70$. 201-3.

4 Grines CL, Browne KF, Marco J, et al. A comparison of immediate angioplasty with thrombolytic therapy for immediate angioplasty with thrombolytic therapy for 673-9.

5 Zijlstra F, de Boer MJ, Hoorntje JCA, Reiffers S, Reiber JHC, Suryapranata H. A comparison of immediate coronary angioplasty with intravenous streptokinase in acute myocardial infarction. $N$ Engl F Med 1993;328:685-91.

6 Gibbons RJ, Holmes DR, Reeder GS, et al. Immediate angioplasty compared with the administration of a thrombolytic agent followed by conservative treatment for myocardial infarction. $N$ Engl $f$ Med 1993;328: $685-91$.

7 International roundup. Primary angioplasty in acute myocardial infarction. Br Heart $\mathcal{F}$ 1995;73:403-16.

8 Gray $\mathrm{HH}$ (on behalf of the British Cardiovascular Intervention Society) Personal communication, 1995.

9 RITA Trial Participants. Coronary angioplasty versus coronary artery bypass surgery: the Randomised Intervention Treatment of Angina (RITA) Trial. Lancet 1993;341 $573-80$

10 Ryan TJ. The critical question of procedure volume minimums for coronary angioplasty. $\mathcal{F} A M A$ 1995;274: 1169-70.

11 Radiation hazards to the cardiologist: Report of a subcommittee of the British Cardiac Society. Br Heart f 1993; 70:489-96.

12 Ritchie JL, Phillips KA, Juft HS. Coronary angioplasty: statewide experience in California. Circulation 1993;88: $2735-43$.

13 Kimmel SE, Berlin JA, Laskey WK. The relationship between coronary angioplasty procedure volume and between coronary angioplasty procedure volum

14 Jollis JG, Peterson ED, De Long ER, et al. The relation between the volume of coronary angioplasty procedures term mortality. N Engl F Med 1994;331:1625-9.

15 International roundup. Surgical cover for angioplasty. $\mathrm{Br}$ Heart $\mathcal{F} 1994 ; 72: 506-8$.

16 Council of the British Cardiovascular Intervention Society. Surgical cover for percutaneous transluminal coronary ngioplasty. Br Heart f 1992;68:339-41.

17 Guidelines for specialist training in cardiology: Report of the Specialist Advisory Committee in Cardiovascula Medicine of the Royal College of Physicians and Counci of the British Cardiac Society. Br Heart $\mathcal{F}$ 1995;73(suppl 1):1-24.

18 Clinical Standards Advisory Group. Access and availability of coronary bypass grafting and coronary angioplasty. HMSO, 1993

19 The Health of the Nation: A strategy for health. HMSO, CM1986.

20 Unger F. European survey on open heart surgery, PTCA and heart catheterisation in 1992. Ann Acad Sci Artium Eur 1993; vol 6.

21 British Cardiac Society: Council statement on the demand and need for cardiac services and the development of a waiting list strategy for cardiac disease. July 1994. 